\title{
Evaluation De La Qualité Physico-Chimique Des Eaux Souterraines Au Voisinage De La Décharge Contrôlée Mohammedia-Benslimane : (Étude Préliminaire)
}

\author{
Ch. Merbouh,
} Laboratoire d'Ecologie et d'Environnement (LEE), Université Hassan IICasablanca, Faculté des Sciences Ben M'Sik, Casablanca, Maroc

\section{K. Belhsaien,}

Laboratoire de Spectroscopie, Modélisation Moléculaire, Matériaux et Environnement (LS3ME), Faculté des Sciences, Université Mohamed V, Av Ibn Battouta, Maroc

\section{A. Zouahri,}

Centre Régional de la Recherche Agronomique de Rabat (INRA), Unité de Recherche sur l'Environnement et la Conservation des Ressources Naturelles, Av Mohamed Belarbi Alaoui,Rabat, Maroc N. Iounes, Laboratoire d'Ecologie et d'Environnement (LEE), Université Hassan IICasablanca, Faculté des Sciences Ben M'Sik, Casablanca, Maroc

\section{Résumé}

L'intérêt de la présente étude est de déterminer la qualité des eaux souterraines au voisinage de la décharge contrôlée de MohammediaBenslimane installée dans la région depuis 2012. Il s'agit des eaux de 7 puits destinées à l'irrigation et/ou à la consommation. Les résultats obtenus sont comparés aux normes marocaines et montrent que les teneurs en chlorures sont très élevées dans tous les puits ( 158.2 à $845.6 \mathrm{mg} / \mathrm{L})$ et dépassent largement

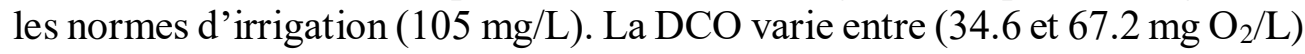
dans cinq puits, ces valeurs sont élevées par rapport à la valeur admissible de l'eau potable ( $25 \mathrm{mg} \mathrm{O}_{2} / \mathrm{L}$ ). Les concentrations enregistrées pour le $\mathrm{Cu}, \mathrm{Fe}$, $\mathrm{Mn}, \mathrm{Zn}$ et Ni correspondent bien aux normes d'irrigation et de potabilité des eaux. Cependant les teneurs en $\mathrm{Pb}$ varient entre $(0.07$ et $0.14 \mathrm{mg} / \mathrm{L})$ dans 4 puits dont 3 sont situés en aval de la décharge. Ces valeurs dépassent celles de la norme de potabilité $(0.05 \mathrm{mg} / \mathrm{L})$. Les concentrations les plus élevées en chrome sont observées dans trois puits situés en amont de la décharge et un à l'aval. En ce qui concerne le cadmium, ses teneurs ne dépassent pas les normes 
de potabilité et d'irrigation, à l'exception d'un puits situé à l'aval de la décharge et dont la valeur dépasse de $(0,001 \mathrm{mg} / \mathrm{L})$ la norme de potabilité. Enfin, la qualité générale des eaux étudiées serait probablement liée aux activités humaines et à la nature du sol traversé par les eaux pluviales et non à la décharge.

Mots clés : Caractérisation Physico-Chimique, ETM, Contamination, Eaux Souterraines, Décharge

\title{
Evaluation of the Physico-Chemical Quality of Groundwater in the Vicinity of the Landfill of Mohammedia-Benslimane: (Preliminary Study)
}

\section{Ch. Merbouh,}

Laboratoire d'Ecologie et d'Environnement (LEE), Université Hassan II-

Casablanca, Faculté des Sciences Ben M'Sik, Casablanca, Maroc

\section{K. Belhsaien,}

Laboratoire de Spectroscopie, Modélisation Moléculaire, Matériaux et Environnement (LS3ME), Faculté des Sciences, Université Mohamed V, Av Ibn Battouta, Maroc

\section{A. Zouahri,}

Centre Régional de la Recherche Agronomique de Rabat (INRA), Unité de

Recherche sur l'Environnement et la Conservation des Ressources

Naturelles, Av Mohamed Belarbi Alaoui,Rabat, Maroc

\section{N. Iounes,}

Laboratoire d'Ecologie et d'Environnement (LEE), Université Hassan II-

Casablanca, Faculté des Sciences Ben M'Sik, Casablanca, Maroc

\begin{abstract}
The aim behind this study is to determine the quality of the groundwater surrounding the landfill that has taken place in MohammediaBenslimane since 2012. It is a matter of seven wells dedicated to irrigation and/or daily use. The results obtained are compared to Moroccan standards and clearly show that the chlorides levels are abnormally high amongst all the wells $(158.2$ to $845.6 \mathrm{mg} / \mathrm{L})$ and go well beyond the irrigation's standards (105 $\mathrm{mg} / \mathrm{L}$ ). The chemical oxygen demand (COD) varies between (34.6 and 67.2 $\mathrm{mg} \mathrm{O}_{2} / \mathrm{L}$ ) in five of the wells: values that exceed the allowed drinking water's
\end{abstract}


value $\left(25 \mathrm{mg} \mathrm{O}_{2} / \mathrm{L}\right)$. The recorded concentrations of the $\mathrm{Cu}, \mathrm{Fe}, \mathrm{Mn}, \mathrm{Zn}$ and the $\mathrm{Ni}$ are well aligned with the standards of irrigation and drinking water quality. However, the $\mathrm{Pb}$ levels, which exceed the standards of potability $(0.05$ $\mathrm{mg} / \mathrm{L})$ range from $(0.07$ and $0.14 \mathrm{mg} / \mathrm{L})$. The highest chromium concentrations were recorded in three of the wells upstream from the landfill and a single one amongst the ones located downstream. Even though the Cadmium levels are aligned with the standards of potability and irrigation in six of the wells, it remains exceeding the standards of the drinking water quality by $(0.001$ $\mathrm{mg} / \mathrm{L})$. As a conclusion, the overall quality of the studied groundwater would probably be linked to the human activities on the surroundings, as well as to the nature of the ground crossed by the stormwater not to the landfill.

Keywords: Physicochemical Characterization, Trace Metals, Contamination, Groundwater, Landfill

\section{Introduction}

Les ressources naturelles en eau au Maroc sont parmi les plus faibles au monde, leur potentiel est évalué à 22 milliards de $\mathrm{m}^{3}$ par an, soit l'équivalent de $700 \mathrm{~m}^{3} /$ habitant/an (MDCEau, 2016), communément admis comme seuil critique indiquant l'apparition de pénuries et de crise latente d'eau. A cette limitation des ressources en eau, s'ajoute la dégradation de la qualité de l'eau suite à l'augmentation de la pollution (MDCEau, 2014) et aux différents modes d'exploitation de cette ressource.

Du fait que les eaux de surface participent à l'alimentation de la nappe, la qualité de l'eau souterraine est directement liée à celle des premières. Par ailleurs, les activités humaines exercent de nombreuses pressions polluantes, d'abord sur les eaux de surface, puis de manière différée sur les eaux souterraines. Ainsi, l'altération de la qualité des eaux souterraines pourrait donc être attribuée à une pollution ponctuelle ou diffuse émanant, entre autres des déchets d'élevage et des eaux usées ou de lixiviats qui ne font pas l'objet de traitement préalable (El Guamri et al., 2007 ; Fekri, 2007 ; Boutayeb et al., 2012 ; Benabbou et al., 2014 ; Idlahcen et al., 2014 ; Laaouan et al., 2016).

L'intérêt de cette étude est de réaliser un diagnostic préliminaire de la qualité des eaux des puits après l'installation de la décharge contrôlée intercommunale de Mohammedia-Benslimane, et de déterminer s'il y a un risque éventuel de leur contamination par les lixiviats issus de cette décharge.

\section{Zone d'étude}

La zone d'étude appartient à la commune territoriale de BenYakhlef relevant de la province de Mohammedia. Le territoire de la commune s'étend sur une superficie d'environ $26 \mathrm{~km}^{2}$ et est limité au Nord par la ville de Mohammedia, au Sud et à l'Est par la ville de Benslimane et à l'Ouest par la 
commune territoriale de Sidi Moussa Majdoub (Monographie communale, 2010).

Le périmètre d'étude est d'environ $12 \mathrm{Km}^{2}$ (Figure 1). Au centre du périmètre se trouve la décharge intercommunale de MohammediaBenslimane. La zone d'étude est délimitée à l'Est par l'Oued Nfifikh et sa forêt et à l'Ouest par la route provinciale P3313.

Selon la prospection de la région, le périmètre d'étude est caractérisé par la présence des dayas temporaires et permanentes et des sols à occupation agricole (Cultures maraichères). C'est une zone rurale, où se trouvent des fermes d'élevage et des pépinières, qui n'abrite pas d'activités industrielles.

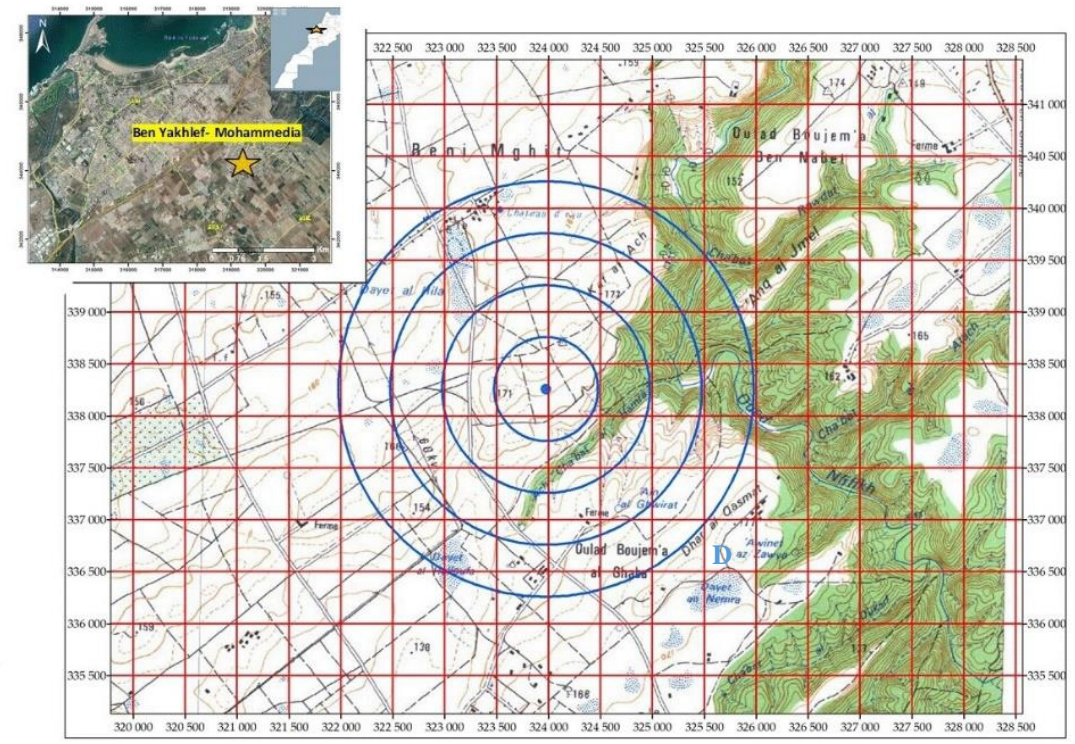

Figure 1. Carte de délimitation de la zone d'étude

La zone d'étude se caractérise par un bioclimat méditerranéen semiaride à hiver doux et humide et à été chaud et sec. La proximité de l'océan atlantique tend à rafraichir la zone en été et à la réchauffer en hiver. La pluviométrie moyenne annuelle au niveau de la commune de Ben Yakhlef est autour de $400 \mathrm{~mm}$ et la température varie entre $10^{\circ} \mathrm{C}$ et $23^{\circ} \mathrm{C}$ (Akil, 1990 ; monographie communale, 2010).

La zone d'étude fait partie du domaine structural de la Meseta côtière marocaine et plus précisément de l'ensemble nord mésetien. La structure géologique de la région montre un socle paléozoïque daté du Cambrien sur lequel se sont déposées alternativement les formations du cambrienordovicien, du permo-trias, du crétacé et du plio-quaternaire. Les affleurements sont généralement constitués de schistes argileux et de pélites rouges avec des intercalations de basaltes dolorétiques (Farki et al., 2012 ; Farki et al., 2014 ; Farki et al., 2016). 
Le réseau hydrographique de la région de Mohammedia est formé par deux Oueds (Oued Nfifikh et Oued El Maleh) et leurs affluents qui traversent la ville en diagonale ainsi que par des dayas permanentes et temporaires.

\section{Matériel et méthodes}

Le choix des sites d'échantillonnage est basé, d'une part sur l'utilisation des eaux souterraines dont la qualité n'est pas connue, et d'autre part sur le sens d'écoulement des eaux et la présence de la décharge contrôlée. Pour déterminer le sens de l'écoulement des eaux souterraines, nous avons effectué des mesures sur le terrain à l'aide d'une sonde piézométrique lumineuse SEBA de type KLL.

La carte piézométrique suivante montre le sens d'écoulement des eaux (SE-NO) et la position des points de prélèvement par rapport à la décharge représentée au centre de la carte par la lettre (D) (figure 2).

L'étude a porté sur sept puits, trois (P1, P2 et P3) sont à l'aval de la décharge et quatre (P4, P5, P6 et P7) sont situés à son amont.

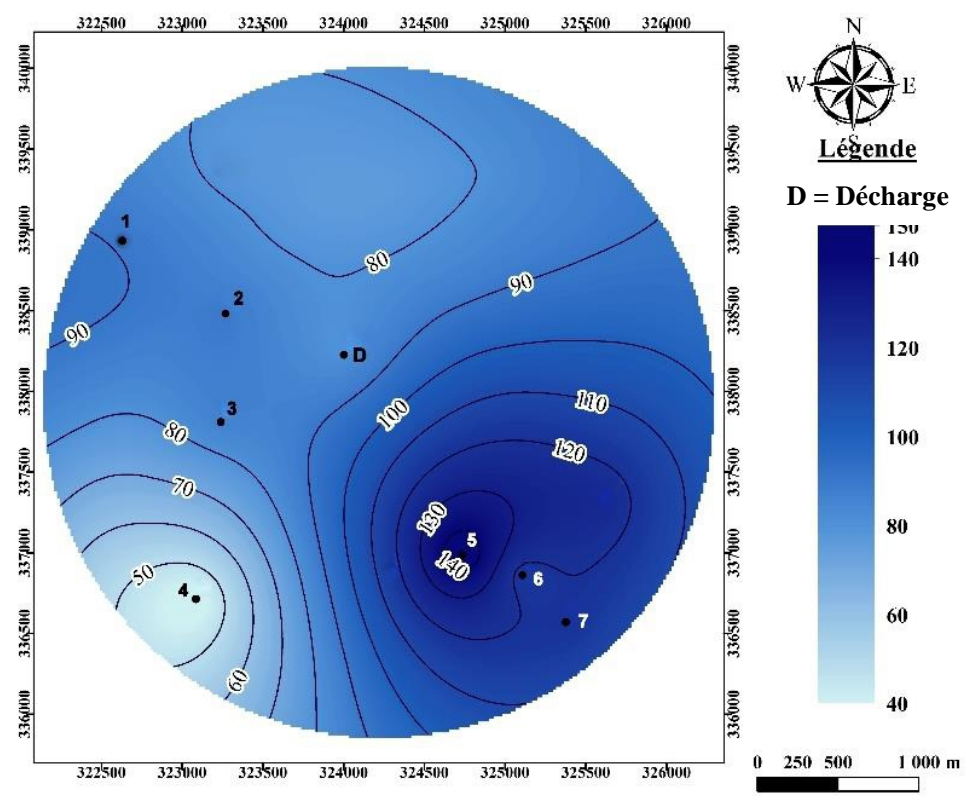

Figure 2. Répartition des puits par rapport au niveau piézométrique

Pour étudier la qualité des eaux de puits, une analyse physico-chimique comportant le $\mathrm{pH}$, la température, la conductivité électrique, l'oxygène dissous, la demande chimique en oxygène (DCO), la demande biologique en oxygène $\left(\mathrm{DBO}_{5}\right)$, la turbidité et les chlorures et une caractérisation de la composition en éléments traces métalliques $(\mathrm{Cd}, \mathrm{Cu}, \mathrm{Cr}, \mathrm{Fe}, \mathrm{Mn}, \mathrm{Ni}, \mathrm{Pb}$, et $\mathrm{Zn)}$ ont été effectuées. 
Les prélèvements des eaux de puits ont été effectués pendant les mois de Mars et Avril 2017 suivant la méthodologie dictée par la NF EN ISO 56671 et sont emportés dans des flacons en polyéthylène, à l'exception des échantillons destinés à l'analyse de l'oxygène dissous. Ces derniers ont été prélevés et mis dans des flacons en verre borosilicaté à bouchons rodés et l'oxygène a été fixé in situ selon la méthode de Winkler. Les échantillons ont été ensuite conservés à $4^{\circ} \mathrm{C}$ dans l'obscurité pendant le transport au laboratoire, puis ont été analysés dans les délais fixés par la norme sus-citée. Les méthodes d'analyses sont celles préconisées par les normes AFNOR et par Rodier (tableau 1).

Tableau 1: Méthodes d'analyses physico-chimiques

\begin{tabular}{|c|c|c|}
\hline Paramètre & Méthode de mesure & Référence \\
\hline Température ${ }^{\circ} \mathrm{C}$ & Thermomètre à mercure & \\
\hline & & $\overline{N F}$ \\
\hline Turbidité NTU & Turbidimètre portatif Version HI 93703, de & \\
\hline & & $50082-1$ \\
\hline$p H$ & $\begin{array}{l}\text { SevenEasy pH- meter S20, de Marque } \\
\text { Mettler-Toledo }\end{array}$ & - \\
\hline C.E $\mathrm{mS} / \mathrm{cm}$ & $\begin{array}{l}\text { Conductivimètre de laboratoire de Marque } \\
\text { ORION, modèle } 162\end{array}$ & - \\
\hline $\mathrm{DBO5} \mathrm{mg} \mathrm{d}^{\prime} \mathrm{O}_{2} / \mathrm{L}$ & Méthode d'OxiTop & $\begin{array}{l}\text { NF EN 1899- } \\
1998\end{array}$ \\
\hline $\mathrm{DCO} \mathrm{mg} \mathrm{d}^{\prime} \mathrm{O}_{2} / \mathrm{L}$ & Méthode à reflux en système ouvert & $\begin{array}{l}\text { NF T90-101- } \\
2001\end{array}$ \\
\hline $\mathrm{O}_{2}$ dissous $\mathrm{mg} / \mathrm{L}$ & $\begin{array}{l}\text { Méthode iodomètrique de Winkler modifiée } \\
\text { CEN }\end{array}$ & $\begin{array}{l}\text { ISO } \\
5813: 1983\end{array}$ \\
\hline Chlorures $\mathrm{mg} / \mathrm{L}$ & Méthode volumétrique de Mohr & Rodier, 1984 \\
\hline
\end{tabular}

\section{Résultats et discussions}

Les résultats des paramètres physico-chimiques étudiés sont représentés dans le tableau 2 et ils sont comparés aux normes marocaines de qualité d'irrigation et de potabilité. 
Tableau 2. Résultats des paramètres physico-chimiques

\begin{tabular}{|c|c|c|c|c|c|c|c|c|}
\hline $\begin{array}{c}\text { Points de } \\
\text { prélèvement }\end{array}$ & $p H$ & $\begin{array}{l}\text { Température } \\
\left({ }^{\circ} \mathrm{C}\right)\end{array}$ & $\begin{array}{c}\text { Turbidité } \\
\text { (NTU) }\end{array}$ & $\begin{array}{c}C . E \\
(\mathrm{mS} / \mathrm{cm})\end{array}$ & $\begin{array}{c}\text { Chlorures } \\
(\mathrm{mg} / \mathrm{L})\end{array}$ & $\begin{array}{c}\mathrm{O}_{2} \\
\text { dissous } \\
(\mathrm{mg} \\
\left.\mathrm{O}_{2} / \mathrm{L}\right)\end{array}$ & $\begin{array}{r}D C O \\
(m g \\
\left.O_{2} / L\right)\end{array}$ & $\begin{array}{c}D B_{5} \\
\left(m g O_{2} / L\right)\end{array}$ \\
\hline$P 1$ & 8.12 & 20.0 & 2.42 & 2.53 & 841.4 & 8.21 & 11.52 & 2.1 \\
\hline$P 2$ & 7.35 & 12.3 & 4.77 & 1.96 & 840.0 & 7.89 & 39.4 & 8.7 \\
\hline$P 3$ & 7.35 & 19.8 & 2.81 & 2.63 & 845.6 & 7.48 & 38.4 & 2.1 \\
\hline P4 & 7.26 & 12.3 & 8.28 & 1.96 & 595.0 & 7.72 & 67.2 & 4.9 \\
\hline P5 & 8.35 & 18.5 & 2.19 & 1.88 & 406.7 & 4.88 & 34.56 & 10.4 \\
\hline P6 & 8.04 & 18.3 & 2.22 & 1.24 & 290.5 & 6.99 & 48.0 & 7.1 \\
\hline P7 & 8.05 & 18.3 & 0.87 & 0.83 & 158.2 & 6.10 & 9.6 & 7.6 \\
\hline $\begin{array}{c}\text { Norme } \\
\text { d'irrigation } \\
\text { (S.E.E.E. } \\
2007 \text { a) }\end{array}$ & $\begin{array}{l}6.5- \\
8.4\end{array}$ & 35 & 5 & 12 & 105 & - & - & - \\
\hline $\begin{array}{l}\text { Norme de } \\
\text { potabilité } \\
\text { (S.E.E.E., } \\
2007 \text { b) }\end{array}$ & $\begin{array}{l}6.5- \\
8.5\end{array}$ & 30 & 5 & 2.7 & 750 & 7 & 25 & 100 \\
\hline
\end{tabular}

\section{Température}

La température est un paramètre clé, du fait qu'elle agit de manière déterminante sur les processus chimiques, l'activité bactérienne et l'évaporation des eaux et influence également la répartition des organismes vivants en milieu aquatique (Leynaud, 1974 ; IBGE, 2005).

Les valeurs de température pour les eaux de puits étudiés varient entre 12.3 et $20^{\circ} \mathrm{C}$, et n'excédant pas les normes de qualité d'irrigation $\left(35^{\circ} \mathrm{C}\right)$ et de potabilité $\left(30^{\circ} \mathrm{C}\right)$ (S.E.E.E, $2007 \mathrm{a}$ et b). Tous les puits étudiés, à l'exception des puits 2 et 4 , présentent des températures qui se rapprochent de celles citées dans la littérature. En effet, l'étude de la qualité des eaux souterraines de différents sites dans la province de Tanger, effectuée par (Fakih et al., 2014), a montré une température de $22^{\circ} \mathrm{C}$. El Asslouj et al. (2007) ont trouvé des températures qui varient entre 18 et $22^{\circ} \mathrm{C}$ dans des puits situés dans la communauté de Mzamza. Les puits situés dans la zone rurale de Meknès montrent une température qui varie entre 20 et $21.04^{\circ} \mathrm{C}$ (Belghiti et al., 2013). Nouayti et al. (2015) ont trouvé des températures qui varient entre 15.1 et $49^{\circ} \mathrm{C}$ dans les eaux souterraines du haut bassin de Ziz. 


\section{Potentiel hydrogène $(\mathrm{pH})$}

Le pH est lié à tous les paramètres de qualité de l'eau, il renseigne sur le statut acido-basique et montre la stabilité d'une eau. Des pH extrêmes ont été associés à certains effets sur la santé, dont l'irritation de la peau et des yeux, mais rien n'indique qu'ils aient des effets directs sur la santé. L'effet le plus important du $\mathrm{pH}$ sur la santé est indirect et est lié à l'exposition aux métaux lourds (SC, 2015).

En ce qui concerne les puits étudiés, les valeurs de $\mathrm{pH}$ trouvées varient entre 7.26 et 8.35. Ces valeurs ne dépassent pas les normes de qualité de potabilité, ni celle d'irrigation (S.E.E.E, 2007 a et b). Ainsi, le pH enregistré est légèrement neutre à basique et ne présente aucun danger pour la santé. Nos résultats concordent avec ceux trouvés par Idlahcen et al. (2014), Laaouan et al. (2016) et Belghiti et al. (2013) dans les eaux de puits de Mohammedia, de Dar Bouazza et de la zone rurale de Meknès respectivement.

\section{Conductivité électrique}

La conductivité sert à apprécier la quantité des sels dissous dans l'eau et donne une appréciation sur sa minéralisation. Une conductivité élevée témoigne d'une quantité de sels dissous très importante (Rodier et al., 2009). Des contrastes de conductivité permettent de mettre en évidence des pollutions, des zones de mélanges ou d'infiltration (Réfea, 2001).

La mesure de la conductivité est importante pour évaluer le degré de toxicité des cultures par l'excès de quantité de sels solubles. Cette toxicité se traduit par l'inhibition de l'assimilation des éléments nutritifs nécessaires à la croissance et au développement de la plante cultivée (Hajlaoui et al., 2015).

On remarque que la valeur de la conductivité pour l'ensemble des puits ne dépasse pas les normes de qualité pour les eaux destinées à la consommation $(\mathrm{C} . \mathrm{E}=2,7 \mathrm{mS} / \mathrm{cm})$ ainsi que pour les eaux destinées à l'irrigation (C. E= $12 \mathrm{mS} / \mathrm{cm})$ (S.E.E.E, 2007 a et b).

\section{Turbidité}

La turbidité est une mesure de la limpidité ou de l'opacité relative de l'eau et n'est pas une mesure directe des matières en suspension dans l'eau, mais plutôt une mesure générale de leur effet de diffusion et d'absorption de la lumière (SC, 2012). Les niveaux de turbidité sont une considération importante comme indicateur de changement dans la qualité de l'eau. Des mesures élevées ou une fluctuation des mesures peuvent indiquer un problème de qualité de l'eau (LeChevallier et al., 1981).

La turbidité varie d'un puits à l'autre mais elle reste inférieure à la norme marocaine d'irrigation et de potabilité (5 NTU) (S.E.E.E, 2007 a et b), à l'exception du puits P4 où on enregistre une valeur de 8,28 NTU. Cette valeur élevée pourrait être liée à une contamination du puits par les déchets 
solides stockés dans une fosse à l'intérieur de la ferme où se trouve ce puits. Ces déchets proviennent de l'élevage pratiqué dans cette ferme et sont exposés aux pluies, ce qui entrainerait une percolation des eaux de pluie entrainant les M.E.S vers les eaux souterraines.

\section{Oxygène dissous}

L'oxygène dissous constitue l'un des plus importants paramètres de qualité des eaux dans la mesure où il est indispensable à la vie aquatique, à différentes réactions chimiques et à la dégradation des polluants biodégradables (Réfea, 2001).

Le taux d'oxygène dissous dans les puits $\mathrm{P} 1, \mathrm{P} 2, \mathrm{P} 3$ et $\mathrm{P} 4$ (8.21, 7.89, 7.48 et $\left.7.72 \mathrm{mg} \mathrm{d} \mathrm{O}_{2} / \mathrm{L}\right)$ respectivement dépassent la valeur imposée par la norme de potabilité (S.E.E.E, 2007 b). Rappelons que les eaux de ces puits sont utilisées uniquement pour l'irrigation. La valeur la plus basse est enregistrée au niveau du puits P5, cette teneur peut être expliquée par l'importance de la dégradation de la matière organique par les microorganismes. En effet la $\mathrm{DBO}_{5}$ trouvée dans ce puits est de l'ordre de 10,4 $\mathrm{mg} \mathrm{d}^{\prime} \mathrm{O}_{2} / \mathrm{L}$. Par ailleurs, la concentration en oxygène dissous est fonction de l'utilisation de l'oxygène par l'activité des microorganismes et les processus d'oxydation et de dégradation de la matière organique présente dans l'eau (IBGE, 2005).

\section{Demande chimique en oxygène}

La demande chimique en oxygène est un paramètre indicateur de pollution. C'est la quantité d'oxygène nécessaire à oxyder les matières existantes dans l'eau quelle que soit leur origine organique ou minérale (Rodier et al., 2009).

Les puits P2, P3, P4, P5 et P6 présentent des teneurs élevées de la DCO par rapport à la norme de potabilité de l'eau (S.E.E.E, 2007 b). Les puits (2, 3, 4 et 5) sont destinés uniquement à l'irrigation donc ils ne posent pas de problèmes pour la santé des usagers alors que le P6 est utilisé à la fois pour l'irrigation et la consommation, la contamination de ce puits pourrait être liée à la présence d'une fosse septique et/ou à la présence des déchets domestiques rejetés sur le site.

\section{Demande biologique en oxygène}

La $\mathrm{DBO}_{5}$ correspond à la quantité de dioxygène nécessaire aux microorganismes aérobies de l'eau pour oxyder les matières organiques, dissoutes ou en suspension dans l'eau. En outre, la pollution organique favorise le développement d'organismes pathogènes pour l'Homme (ASEF, 2010). Et ce paramètre constitue un bon indicateur de la teneur en matières organiques biodégradables d'une eau (Rodier et al., 2009). 
Les résultats de tous les puits ne présentent pas de valeurs qui excèdent la norme marocaine de potabilité (S.E.E.E, 2007 b).

La DCO et la DBO sont des paramètres complémentaires. En outre, le rapport $\mathrm{DCO} / \mathrm{DBO}_{5}$ donne une première estimation de la biodégradabilité de la matière organique. Le tableau 3 présente les indices de biodégradabilité des différents puits en fonction du rapport $\mathrm{DCO}^{\mathrm{D}} \mathrm{DBO}_{5}$ selon Rodier et al. (2009).

Tableau 3. Rapport de biodégradabilité

\begin{tabular}{|c|c|c|}
\hline & $\begin{array}{l}\text { RAPPORT } \\
\text { DCO/DBO5 }\end{array}$ & BIODÉGRADABILITÉ \\
\hline P1 & 5.48 & $\begin{array}{l}\text { Difficilement } \\
\text { biodégradable }\end{array}$ \\
\hline $\mathbf{P 2}$ & 4.41 & $\begin{array}{l}\text { Moyennement } \\
\text { biodégradable }\end{array}$ \\
\hline P3 & 18.28 & $\begin{array}{l}\text { Difficilement } \\
\text { biodégradable }\end{array}$ \\
\hline P4 & 13.71 & $\begin{array}{l}\text { Difficilement } \\
\text { biodégradable }\end{array}$ \\
\hline P5 & 0.09 & $\begin{array}{c}\text { Facilement } \\
\text { biodégradable }\end{array}$ \\
\hline P6 & 6.76 & $\begin{array}{l}\text { Difficilement } \\
\text { biodégradable }\end{array}$ \\
\hline P7 & 1.26 & $\begin{array}{c}\text { Facilement } \\
\text { biodégradable }\end{array}$ \\
\hline
\end{tabular}

Les résultats issus des analyses de la $\mathrm{DCO}$ et de la $\mathrm{DBO}_{5}$ comparés à l'indice de biodégradabilité (Rodier et al., 2009) montrent que les puits 5 et 7 sont riches en matière organique fortement biodégradable. Les puits (P1, P3, $\mathrm{P} 4$ et $\mathrm{P} 6$ ) dont le rapport $\mathrm{DCO} / \mathrm{DBO}$ est supérieur à 5, présentent une faible biodégradabilité.

\section{Chlorures}

Les chlorures sont des anions inorganiques importants contenus en concentrations variables dans les eaux naturelles, généralement sous forme de chlorure de sodium $(\mathrm{NaCl})$ et de potassium $(\mathrm{KCl})$. Ils sont souvent utilisés comme indice de pollution (Lakhili et al., 2015).

Les résultats obtenus montrent que les teneurs en chlorures sont très élevées dans tous les puits et dépassent largement les normes d'irrigation (105 $\mathrm{mg} / \mathrm{L}$ ) (S.E.E.E, 2007 a). Les eaux de ces puits sont utilisées dans l'irrigation et ceci ne serait pas sans impact sur le rendement des cultures par des effets de toxicité dus à un stress salin ou à un stress oxydatif (Mezni et al., 1999 ; Lepengue et al., 2010 ; R'him et al., 2013, Hajlaoui et al., 2015).

La présence des chlorures à ces taux dans tous les puits est probablement liée à la nature du sol traversé par les eaux de pluie. D'après Leveque (2009), les formations post-primaires (crétacé et plio-quaternaire) du site constituent une couverture perméable, facilitant ainsi l'infiltration et 
l'enrichissement des terrains triasiques par différents indicateurs facilement mobiles tels que les chlorures.

\section{Eléments traces métalliques}

Cette partie du travail s'intéresse aux éléments traces métalliques présents dans les eaux de puits destinées à l'irrigation et/ou à la consommation. La figure 3 illustre les valeurs trouvées des éléments traces métalliques dans les eaux de puits étudiés :
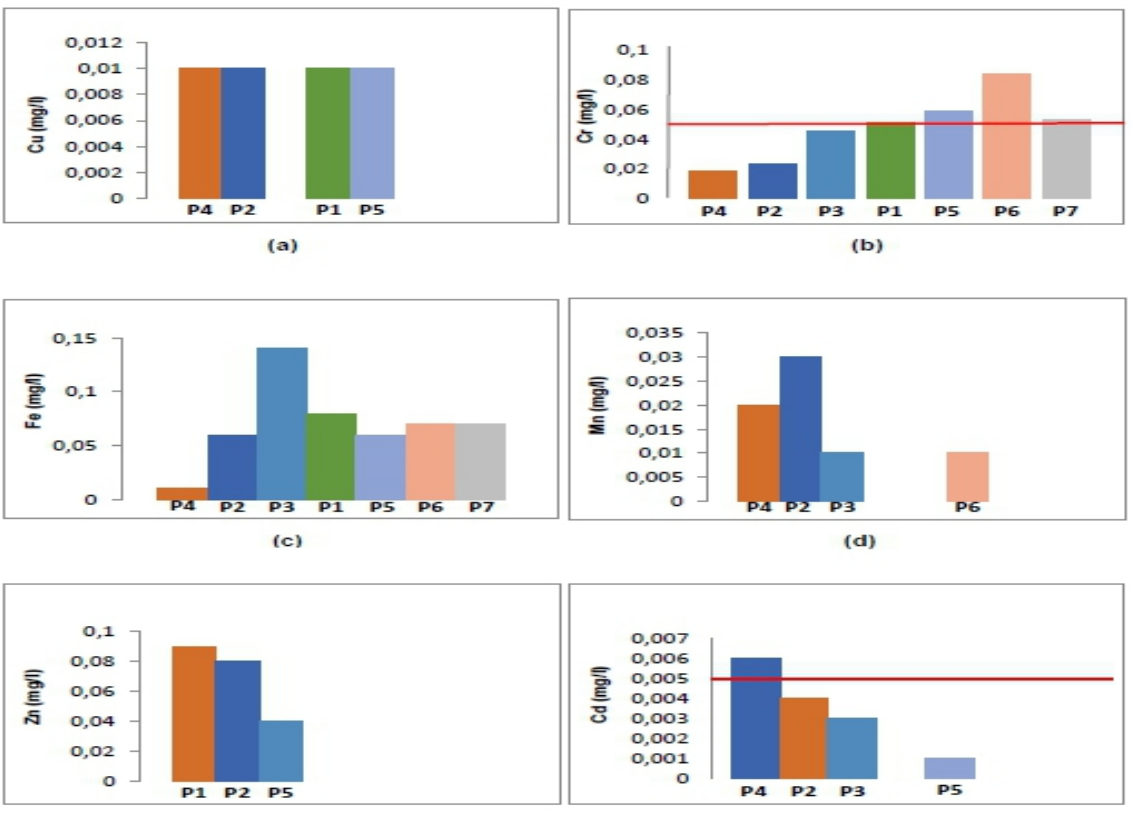

(e)
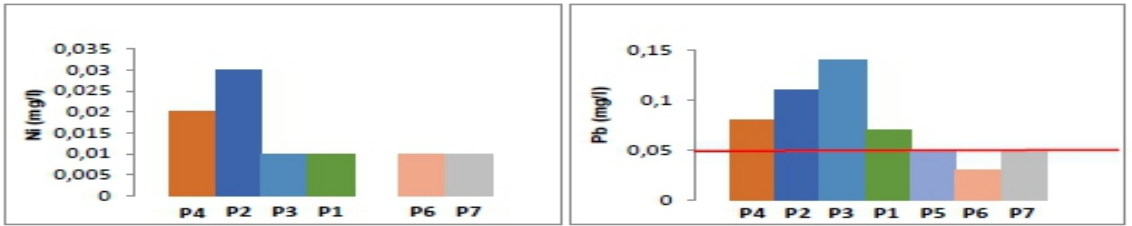

(g)

(h)

Figure 3. Concentration en éléments traces métalliques dans les eaux de puits : (a)

Cuivre (Cu) ; (b) Chrome (Cr) ; (c) Fer (Fe) ; (d) Manganèse (Mn) ; (e) Zinc (Zn) ; (f) Cadmium (Cd); (g) Nickel (Ni); (h) Plomb (Pb).

Les teneurs en Fer et en Mn varient entre (0.01 et $0.14 \mathrm{mg} / \mathrm{L}$ ) et entre ( 0 et $0,03 \mathrm{mg} / \mathrm{L}$ ) respectivement. Ces valeurs sont dans les normes d'irrigation et de potabilité des eaux (S.E.E.E, 2007 a et b). Les effluents industriels, les eaux usées ainsi que les lixiviats provenant des sites d'enfouissement des déchets peuvent contribuer à la présence de fer et de manganèse dans les eaux souterraines (Belle, 2008). La présence de ces faibles quantités serait liée à la nature du sol, aux eaux usées et aux déchets domestiques. 
Les valeurs de zinc enregistrées dans les puits 2, 3 et 4 sont de l'ordre de $0.08 \mathrm{mg} / \mathrm{L}, 0.04 \mathrm{mg} / \mathrm{L}$ et $0.09 \mathrm{mg} / \mathrm{L}$ respectivement, et restent dans les normes d'irrigation et de potabilité des eaux (S.E.E.E, 2007 a et b). Par ailleurs, le Zn n'a pas été détecté dans les puits 1, 5, 6 et 7. La présence du zinc peut être liée à l'utilisation des produits phytosanitaires (Desbordes, 2000).

Les teneurs enregistrées en cadmium pour les eaux des puits analysées ne dépassent pas les normes de potabilité et ceux d'irrigation (S.E.E.E, 2007 a et b), à l'exception du puits 4 dont la teneur du Cd dépasse de $0,001 \mathrm{mg} / \mathrm{L}$ la norme de potabilité. Ceci dit, cela ne risque pas de nuire directement à la santé humaine car les eaux de ce puits sont utilisées uniquement pour l'irrigation. Le cadmium peut être entrainé par les pluies à partir des fumées industrielles des établissements implantés dans la région, comme il peut provenir des engrais utilisés en agriculture (Desbordes, 2000).

Les teneurs détectées en nickel varient entre ( 0 et $0.003 \mathrm{mg} / \mathrm{L})$ dans les puits faisant l'objet de l'étude et restent très en dessous des normes marocaines de potabilité et d'irrigation (S.E.E.E, 2007 a et b). La présence de ce métal dans les eaux souterraines peut être liée aux activités humaines (Desbordes, 2000).

Les puits $\mathrm{P} 1, \mathrm{P} 2, \mathrm{P} 3$ et $\mathrm{P} 4$ présentent des concentrations en plomb variant entre $(0.08$ à $0.14 \mathrm{mg} / \mathrm{L})$. Ces valeurs dépassent les normes de qualité des eaux potables mais restent très en dessous des normes d'irrigation. La présence du plomb dans ces eaux peut être liée à la pollution agricole.

\section{Conclusion}

Les résultats de cette étude fournissent une première description de la qualité des eaux des puits dans la région de Ben Yakhlef après l'installation de la décharge contrôlée intercommunale de Mohammedia-Benslimane. Ces eaux sont utilisées par la population pour l'irrigation, la consommation et pour les usages domestiques. Elles connaissent une pollution organique importante caractérisée par des valeurs élevées de la DCO allant jusqu'à $67.2 \mathrm{mg} \mathrm{O} / 2$ et la turbidité allant à 8.28 NTU, et une charge minérale traduite par une concentration importante en chlorures $(845.6 \mathrm{mg} / \mathrm{L})$. La pollution par les métaux lourds est aussi présente $(\mathrm{Pb}, \mathrm{Cd}$, et $\mathrm{Cr})$. Les teneurs trouvées semblent être liées à une potentielle intrusion de pollutions phytosanitaire et domestique, à des émissions industrielles régionales et éventuellement à une pollution naturelle liée à la nature du sol traversé par les eaux pluviales. Comme les puits situés à l'amont de la décharge se trouvent également contaminés, cela permet de conclure que cette pollution n'est pas liée à la décharge. En outre, cette décharge est un centre d'enfouissement technique qui est construit dans le but d'éviter toute contamination de la nappe par ses lixiviats qui sont récoltés et drainés vers une station de traitement du centre. 
Enfin, les autorités dans la gestion des ressources en eau, puissent trouver dans ce travail un moyen attisant leurs compétentes afin de contrôler l'utilisation rationnelle des produits phytosanitaires dans la région, l'installation de réseaux d'assainissement des eaux usées sans oublier le suivi et le contrôle de la décharge.

\section{References:}

1. Akil M. (1990). Les dépôts quaternaires littoraux entre Casablanca et Cap Beddouza (Méséta côtière marocaine) - Etude géomorphologique et sédimentologique. D.E.S., troisième cycle de géologie. Faculté des Sciences de Rabat, université Mohammed V. 137 p.

2. ASEF (Association Santé Environnement France) (2010). Pollution de l'environnement : Origines et impacts. Synthèse. Pp 1-7. [En ligne]. Disponible http://doc.hubsante.org/doc_num.php?explnum_id=9366 [Consulté le 18 Oct. 2018].

3. Belghiti M.L., Chahlaoui A., Bengoumi D., El Moustaine R. (2013). Etude de la qualité physico-chimique et bactériologique des eaux souterraines de la nappe plio-quaternaire dans la région de Meknès (Maroc). Larhyss Journal, ISSN 1112-3680, n¹4, Juin 2013, p 21-36.

4. Belle E. (2008). Évolution de l'impact environnemental de lixiviats d'ordures ménagères sur les eaux superficielles et souterraines, approche hydrobiologique et hydrogéologique. Site d'étude : décharge d'Étueffont (Territoire de Belfort - France). Hydrologie. Université de Franche-Comté, 2008. Français. <tel-00400680>.

5. Benabbou M., Elhaji M., Zemzami M., Fadil F. (2014). Impact des lixiviats de la décharge sauvage de la ville de Taza sur les ressources hydriques (Maroc). Afrique Science ISSN (1813-548X). pp.171-180.

6. Benaissa A. (2012). Etude de la faisabilité d'élimination de certains colorants textiles par certains matériaux déchets d'origine naturelle. Mémoire de Master : Sciences Séparatives et Environnement. FSTlemcen. Université Abou Bakr Balkaid.

7. Boutayeb M., Bouzidi A., Fakhaoue M. (2012). Etude de la qualité physico-chimique des eaux usées brutes de cinq villes de la région de la Chaouia Ouardigha (Maroc). Bulletin de l'institut scientifique, Rabat, section Sciences de la vie, n³4 (2), p. 145-150.

8. Desbordes A. (2000). Pollutions des eaux souterraines en Picardie. Mémoire. Maîtrise BG, Fac. Sciences, Amiens, 50 p.

9. El Asslouj J., Kholtei S., El Amrani N., Hilali A. (2007). Analyse de la qualité physico-chimique des eaux souterraines de la communauté des Mzamza, au voisinage des eaux usées. Afrique SCIENCE 03(1) (2007) $109-122$. 
10. El Guamri Y., Belghyti D., Cisse M., El Kharrim K., Sylla I., Raweh S., Barkia H., Hassouni T., Jamber A. (2007). Etude physico-chimique et parasitologique des eaux usées destinées à l'irrigation du périmètre périurbain de Fouarat (Kenitra, Maroc). Agronomie Africaine 19 (3) : 251-261.

11. Fakih Lanjri A., Brigui J., Elcadi A., Khaddor M., Salmoune F. (2014). Caractérisation physico-chimique et bactériologique des eaux souterraines de Tanger. Journal of Materials and Environment Science (ISSN: 2028-2508), pp. 2230-2235.

12. Farki K., Zahour G., Baroudi Z., Alikouss S., Zerhouni Y., El Hadi H., Darhnani M. (2016). Mines et carrières triasico-liasiques de la région de Mohammedia : inventaire, valorisation et étude d'impact environnemental. International Journal of Innovation and Scientific Research, Vol. 20 No. 2, pp. 306-326.

13. Farki K., Zahour G., El Hadi H., Alikouss S., Zerhouni Y. (2014). Les tholéiites fini-triasiques de Mohammedia (meseta côtière, Maroc) : Témoins d'un volcanisme de Rift intracontinental avorté. European Scientific Journal. July 2014 edition. Vol. 10, No. 20.

14. Farki K., Zahour G., Zerhouni Y., Hamid W. (2012). Contribution à la compréhension de l'évolution sédimentaire et tectono-volcanique de la série Triasico-Liasique de l'Oued Nfifikh (meseta côtière, Maroc). Ann. Soc. Géol. Du Nord. T. 19 (2éme série), p. 1-12.

15. Fekri A. (2007). Impact de la décharge de Médiouna sur les ressources en eaux souterraines. Doctorat d'état en géologie. Faculté des sciences Ben M'Sik - Université Hassan II.

16. Hajlaoui H., Maatallah S., Denden M. (2015). Effet du stress salin sur l'efficience d'utilisation d'azote et les bilans ioniques chez deux variétés de maïs (Zeamays L.) fourragère. Journal of Animal \& Plant Sciences, 2015. Vol.24, Issue 3 : 3787-3801.

17. IBGE (Institut Bruxellois pour la Gestion de l'Environnement) (2005). Qualité physico-chimique et chimique des eaux de surface : Cadre Général. Publication/Fiche documentée. Observatoire des données de l'environnement Novembre 2005. Pp 1-16.

18. Idlahcen A., Souabi S., Taleb A., Zahidi K., Bouezmarni M. (2014). Evaluation de la pollution générée par les lixiviats de la décharge publique de la ville de Mohammedia et son impact sur la qualité des eaux souterraines. ALMA MATER Publishing House 'VASILE ALECSANDRI' University of Bacau (ISSN 1582-540X), pp.035-050.

19. Laaouan M., Aboulhassan M.A., Bengamra S., Taleb A., Souabi S., Tahiri M. (2016). Etude comparative de la contamination des eaux souterraines des villes de Mohammedia, Temara et Dar Bouazza par 
les nitrates (Meseta marocaine). Journal of Materials and Environmental Science (ISSN : 2028-2508), pp.1298-1309.

20. Lakhili F., Benabdelhadi M., Bouderka H., Lahrach H., Lahrach A. (2015). Etude de la qualité physico-chimique et de la contamination métallique des eaux de surface du bassin versant de Beht (Maroc). European Scientific Journal, N¹1. Pp. 132-147.

21. LeChevallier M.W., Evans J.M., Selder R.J. (1981). Effect of turbidity on chlorination efficiency and bacterial persistence in drinking water. Applied and Environmental Microbiology. Vol. 42 No. 1. P. 159-167.

22. Lepengue A. N., Mouaragadja I., M’batchi B., Ake S. (2010). Effet du chlorure de sodium $(\mathrm{NaCl})$ sur la germination et la croissance du maïs (ZeamaysL., Poaceae) au Gabon. International Journal of Biological and Chemical Sciences. 4(5): 1602-1609.

23. Leynaud G., Allardi J., Jamain R., Rogger M., Savary M. (1974). Incidences d'un rejet thermique en milieu fluvial sur les mouvements des populations ichtyologiques. Bulletin Français de Pisciculture, (255) (1974) 41-50.

24. Leveque N. (2009). Synthèse des premiers éléments de création d'un réseau de gestionnaires de zones humides littorales en vue d'échanges d'expériences. Rapport de stage. UPVM III.

25. Matini L., Moutou J.M., Kongo-Mantono M.S. (2009). Evaluation hydro-chimique des eaux souterraines en milieu urbain au sud-ouest de Brazzaville, Congo. Afrique science. ISSN 1813-548X. Pp 82-98.

26. MDCEau (Ministère délégué chargé de l'eau) (2016). Ressource en eau : présentation générale [en ligne]. Disponible sur www.water.gov.ma/ressources-en-eau/presentation-generale/

[Consulté le 21 mai 2018].

27. MDCEau (Ministère Délégué Chargé de l'Eau) (2014). Préservation de la qualité des ressources en eau et lutte contre la pollution : Valeurs limites de Rejet à respecter par les déversements (Normes de pollution). Service Contrôle de la pollution de l'Eau, Rabat.

28. Mezni M., Bizid E., Hamza M. (1999). Effets de la salinité des eaux d'irrigation sur la survie et la croissance de trois cultivars de luzerne pérenne. Fourrage 158, 169-178.

29. Monographie communale (2010). Commune rurale de BniYakhlef. Préfecture de Mohammedia. Hydraumet.

30. NF EN ISO 5667-1 (Mars 2007). Qualité de l'eau - Echantillonnage Partie 1 : lignes directrices pour la conception des programmes et des techniques d'échantillonnage (Indice de classement : T90-511-1).

31. Nouayti N., Khattach D., Hilali M. (2015). Evaluation de la qualité physico-chimique des eaux souterraines des nappes du Jurassique du 
haut bassin de Ziz (Haut Atlas central, Maroc). J. Mater. Environ. Sci. 6 (4), 1068-1081.

32. Réfea (REseau Francophone sur l'Eau et l'Assainissement) (2001). Fiche d'analyse physico-chimique [en ligne]. Disponible sur https://www.oieau.org/ReFEA/fiches/AnalyseEau/Physico_chimie_P resGen.htm [Consulté le 13 Aout 2018].

33. R'him T., Tlili I., Hnan I., Ilahy R., Benali A., Jebari H. (2013). Effet du stress salin sur le comportement physiologique et métabolique de trois variétés de piment (Capsicum annuum 1.). Journal of Applied Biosciences 66:5060 - 5069.

34. Rodier J., Legube B., Merlet N., Coll. (2009). L’analyse de l'eau. 9éme Edition, Dunod, Paris, 1579 p.

35. SC (Santé Canada) (2015). Recommandations pour la qualité de l'eau potable au Canada : Document technique-Le $\mathrm{pH}$. Bureau de la qualité de l'eau et de l'air, Direction générale de la santé environnementale et de la sécurité des consommateurs, Santé Canada, Ottawa (Ontario). (Numéro de catalogue H14428/2016 F-PDF).

36. SC (Santé Canada) (2012). Recommandations pour la qualité de l'eau potable au Canada : Document technique- La turbidité. Bureau de la qualité de l'eau et de l'air, Direction générale de la santé environnementale et de la sécurité des consommateurs, Santé Canada, Ottawa (Ontario). (Numéro de catalogue H144-9/2013F-PDF).

37. S.E.E.E (Secrétariat d'État de l'Eau et de l'Environnement) (2007) a. Normes de qualité : Eaux destinées à l'irrigation. Secrétariat d'Etat auprès du Ministère de l'Energie des Mines, de l'Eau et de l'Environnement, chargé de l'Eau et de l'Environnement. Rabat.

38. S.E.E.E (Secrétariat d'État de l'Eau et de l'Environnement) (2007) b. Normes de qualité : Eaux utilisées pour la production de l'eau potable. Secrétariat d'Etat auprès du Ministère de l'Energie des Mines, de l'Eau et de l'Environnement, chargé de l'Eau et de l'Environnement. Rabat. 